\title{
BMJ Open Evaluation of plasma microRNA-122, high-mobility group box 1 and keratin-18 concentrations to stratify acute gallstone disease: a pilot observational cohort study in an emergency general surgery unit
}

Francesca Th'ng, ${ }^{1}$ Bastiaan Vliegenthart, ${ }^{2}$ Jonathan D Lea, ${ }^{3}$ Daniel J Antoine, ${ }^{3}$ James W Dear, ${ }^{2}$ Damian J Mole, ${ }^{1,4,5}$ Edinburgh Emergency Surgery Study Group, Royal Infirmary of Edinburgh, NHS Lothian

To cite: Th'ng F, Vliegenthart B, Lea JD, et al. Evaluation of plasma microRNA-122, highmobility group box 1 and keratin-18 concentrations to stratify acute gallstone disease: a pilot observational cohort study in an emergency general surgery unit. BMJ Open 2018;8:e020061. doi:10.1136/ bmjopen-2017-020061

- Prepublication history and additional material for this paper are available online. To view these files, please visit the journal online (http://dx.doi. org/10.1136/bmjopen-2017020061).

International Surgical Congress of the Association of Surgeons of Great Britain and Ireland (ASGBI) 2016, Belfast, Ireland. Oral presentation as Short Paper. Basic and Applied Clinical Science session, on 11 May 2016. Presentation number 370 . Abstract published in British Journal of Surgery: 'The role of microRNA biomarkers to predict complications of gallstones' (Aug 2016. Vol. 103, Issue S6, Page 17).

Received 12 0ctober 2017 Revised 8 March 2018 Accepted 23 March 2018

Check for updates

For numbered affiliations see end of article.

Correspondence to Miss Francesca Th'ng; fcsthng1@doctors.org.uk

\section{ABSTRACT}

Objective To obtain pilot data to evaluate the discriminatory power of biomarkers microRNA-122 (miR122), high-mobility group box 1 (HMGB1), full-length keratin-18 (flk-18) and caspase-cleaved keratin-18 (cck18) in plasma to identify potential biliary complications that may require acute intervention.

Design An observational biomarker cohort pilot study. Setting In a Scottish University teaching hospital for 12 months beginning on 3 September 2014.

Participants Blood samples were collected from adults ( $\geq 16$ years old) referred with acute biliary-type symptoms who have presented to hospital within 24 hours prior were recruited. Patients unable or refused to give informed consent or were transferred from a hospital outside the National Health Service regional trust were excluded. Primary outcome measures To evaluate whether circulating miR-122, HMGB1, flk-18 and cck-18 can discriminate between people with and without gallstone disease and uncomplicated from complicated gallstone disease during the first 24 hours of hospital admission. Results 300 patients were screened of which 285 patients were included. Plasma miR-122, cck-18 and flk-18 concentrations were increased in patients with gallstones compared with those without (miR-122: median: $2.89 \times 10^{4}$ copies $/ \mathrm{mL}$ vs $0.90 \times 10^{4}$ copies $/ \mathrm{mL}$ $(p<0.001)$; $c c k-18: 121.2 \mathrm{U} / \mathrm{L}$ vs $103.5 \mathrm{U} / \mathrm{L}(p=0.031)$; flk-18: 252.4U/L vs 145.1U/L ( $p<0.001))$. Uncomplicated gallstone disease was associated with higher miR-122 and cck-18 concentrations than complicated disease (miR-122: $5.72 \times 10^{4}$ copies $/ \mathrm{mL}$ vs $2.26 \times 10^{4}$ copies $/ \mathrm{mL}(\mathrm{p}=0.023)$; cck-18: $139.7 \mathrm{U} / \mathrm{L}$ vs $113.6 \mathrm{U} / \mathrm{L}(\mathrm{p}=0.047))$. There was no significant difference in HMGB1 concentration between patients with and without gallstones $(p=0.559)$. Separation between groups for all biomarkers was modest.

Conclusion miR-122 and keratin-18 plasma concentrations are elevated in patients with gallstones. However, this result is confounded by the association between biomarker concentrations, age and gender. In this pilot study, miR-122 and keratin-18 were not sufficiently
Strengths and limitations of this study

- Laboratory analyses were carried out blinded to clinical data.

- The study only included patients who had presented to hospital within 24 hours, thus ensuring that biomarker concentration levels would be as closely representative of the patients' presenting condition as possible.

- Sample size of study is relatively small.

- Serial patient blood sampling for biomarker analyses may have been useful to determine the true nature of biomarker concentration changes as the biliary diseases progress.

microRNA-122 and keratin-18 plasma concentrations are confounded by age and gender.

discriminatory to be progressed as clinically useful biomarkers in this context.

\section{INTRODUCTION}

Gallstone disease is one of the most common reasons for persons to present to hospital with gastrointestinal disease in Western countries. ${ }^{1}$ In the UK, there are approximately 330 people per 100000 adult population admitted with an acute biliary disease annually. $^{2}$ A point-of-care diagnostic test that can stratify patients with acute biliary-type symptoms according to their need for further investigation or intervention will be advantageous to patients and has the potential to reduce healthcare expenditure. Individuals with uncomplicated biliary colic and aseptic choledocholithiasis may be safely discharged without the need for inpatient management 
and can be managed in an outpatient or day-case setting. Conversely, patients with complications, for example, cholecystitis, cholangitis and gallstone pancreatitis are best managed as inpatients according to current national standards.

Standard available clinicopathological data for patients presenting acutely with gallstones in the emergency setting include clinical history and examination, routine laboratory results and ultrasound scan (USS) or MRI findings. ${ }^{3}$ Currently, this set of investigations cannot always identify whether patients have complications of gallstones that necessitate admission. Therefore, a biomarker panel that could be used as an adjunct to existing investigations to guide treatment decisions would be extremely advantageous.

microRNA-122 (miR-122), high-mobility group box 1 (HMGB1), full-length keratin-18 (flk-18) and caspasecleaved keratin-18 (cck-18) have been identified as early acute hepatic injury biomarkers at presentation to hospital, particularly in the context of paracetamol overdose. ${ }^{45}$ miRs are single stranded, small non-protein-coding RNAs. miR-122 is expressed near exclusively in the hepatocyte and provides enhanced hepatic specificity over all current liver injury biomarkers. ${ }^{6}$ miR-122 is important in the regulation of cholesterol and fatty acid metabolism; these mechanisms are key contributors to the pathogenesis of gallstones. ${ }^{78}$ HMGB1 is a nuclear-binding protein with proinflammatory activity that is released by immune and necrotic cells. ${ }^{9}{ }^{10}$ Keratins are part of the intermediate filament system and they play an important role in cellular mechanisms. They are a hallmark of epithelial cells, including hepatocytes and biliary cells. ${ }^{11} 12$ During apoptosis, flk-18 is cleaved by caspases to produce the fragment cck- $18 .{ }^{13}$ flk-18 is released into blood during necrosis and cck-18 during apoptosis. ${ }^{14}$ Although these biomarkers (particularly miR-122) are enriched specifically in hepatocyte, studies in preclinical models have demonstrated increased circulating miR-122, HMGB1 and keratin-18 following bile duct ligation, ${ }^{15}$ which may reflect subsequent hepatocyte injury. In humans with cholecystitis, keratin-18 has been reported to be elevated in both bile and serum samples, when compared with a control group of healthy volunteers. ${ }^{16}$ Shifeng et at ${ }^{6}$ reported that circulating miR-122 has a high diagnostic value with regard to distinguishing patients with biliary calculi from normal controls. In that study, the area under the receiver operator curve) was 0.93 , which suggests very high accuracy. However, the controls were healthy subjects, whereas in clinical practice, the relevant comparison group will be patients with other aetiologies of abdominal pain. In the present study, we recruited a cohort of patients with acute abdominal pain.

The aim of this pilot study was to obtain sufficient data to make a go/no-go decision regarding progression to a larger scale study to evaluate whether circulating hepatic injury biomarkers discriminate between people with and without gallstone disease and uncomplicated from complicated gallstone disease during the first 24 hours of hospital admission.

\section{METHODS}

Patient and public involvement

The research question and outcome measures developed as a result of the high incidence of gallstone diseases and the clinical significance they have on patients' health and quality of life. Patients were only involved as study participants and not in any other aspects of the study. Patient advisers also include those listed as the Study Contributors.

\section{Study design and setting}

This pilot study was an observational cohort biomarker study conducted in the Emergency Department and Emergency General Surgery Department at the Royal Infirmary of Edinburgh, National Health Service (NHS) Lothian, UK.

\section{Inclusion and exclusion criteria}

All adult patients referred to the on-call general surgeons with acute abdominal pain, irrespective of the characteristics of the pain and with a differential diagnosis of gallstone disease on index presentation were eligible for inclusion. Patients must have had presented to hospital within 24 hours prior to recruitment. Patients who were unable to give informed consent, or who refused to participate in the study, or who were younger than 16 years of age or transferred from a hospital outside the NHS regional trust (NHS Lothian) were excluded from the study.

\section{Study population, sample size and data collection}

This proof-of-concept research study was designed to inform a power calculation for a multicentre qualification study, and therefore the sample size was pragmatic. This study had been approved to recruit a total of 300 patients over a period of 2 years.

Recruitment was for 52 weeks: 3 September 2014 to 2 September 2015. Potential participants were identified at the on-call general surgery team's daily handover. All patients recruited to the study had given informed consent. Clinical details, imaging reports, operation findings and pathology reports were obtained from handwritten and electronic medical notes. Each patient was then followed up through the electronic record for a minimum of 3 months. Whole blood was sampled within 24 hours of hospital presentation into Ethylenediaminetetraacetic acid (EDTA) vacutainer tubes (S-Monovette, Sarstedt), centrifuged at $1000 \mathrm{xg}$ for $15 \mathrm{~min}$ at $4^{\circ} \mathrm{C}$ and the plasma layer retained. The supernatant was then separated into aliquots and stored at $-80^{\circ} \mathrm{C}$ until analysis.

\section{Variables and definitions}

Clinical data included patient demographics, routine laboratory blood and microbiology results, imaging investigations, and/or information on surgical interventions 
and pathology results. The final diagnoses of the patients were determined by histopathology results, operative findings, imaging investigations and/or discharge scripts, following that hierarchy. Patients with a diagnosis of non-specific abdominal pain (NSAP) had normal investigation results and a definitive cause of their abdominal pain was not found. All patients with NSAP had underwent imaging with USS and/or magnetic resonance cholangiopancreatography (MRCP). Patients with gallstone diseases were grouped into 'uncomplicated' and 'complicated' categories: patients with 'uncomplicated' disease had either biliary colic (cholelithiasis in gallbladder) or aseptic choledocholithiasis (choledocholithiasis without clinical, laboratory or microbiological evidence of sepsis); patients with 'complicated' gallstone disease were defined as patients with significant clinical, laboratory or pathological inflammation or infection, which included those with cholangitis (septic choledocholithiasis), cholecystitis or gallstone pancreatitis. Patients with 'other' diseases included patients with any other non-gallstone-related disease that was diagnosed by a clinical (eg, urinalysis), imaging or invasive (eg, endoscopy) investigation. Each specific diagnosis is given in the flow chart (figure 1).

\section{Total RNA extraction, quantitative real-time PCR and ELISA analysis}

Laboratory analyses of all biomarkers were carried out blinded to participant clinical data. Analysis of miR-122 was carried out in the Centre for Cardiovascular Sciences, University of Edinburgh); HMGB1, flk-18 and cck-18 laboratory analyses were carried out at the MRC Centre for Drug Safety Science, University of Liverpool.

miR was extracted from plasma using the miRNeasy Serum/Plasma Kit (Qiagen, Venlo, Netherlands) according to the manufacturer's protocol. ${ }^{17}{ }^{18}$ Synthetic miR-39 (at $1.6 \times 10^{8}$ copies $/ \mu \mathrm{L}$ ) was spiked in as an internal control. miRs were measured with Taqman-based quantitative PCR. Small RNA elutes were reverse transcribed using specific stem-loop reverse-transcription RT primers (Applied Biosystems, Foster City, California, USA) for each target miR species, following the manufacturer's instructions. Specific stemloop rt primer targeting UGGAGUG UGACAAUGGUGUUUG and 5' UCACGGGGUGU AAAUCAGCUUG 3' was used. No template controls were included to test for miR contamination. The expressions of miR-39 and miR-122 were analysed using the standard 2-dct method ${ }^{19}$ normalised to the miR-39 spiked internal control.

Plasma HMGB1 and keratin-18 were determined by ELISA according to the manufacturer's guidelines (Shino-Test/ IBL International ${ }^{20} 21$ for HMGB1; and PEVIVA ${ }^{22}$ for cck-18 and flk-18) and our previously published protocols. ${ }^{523}$

\section{Statistical analysis}

Continuous variables are presented as medians and IQR. Categorical variables are presented as frequencies. The

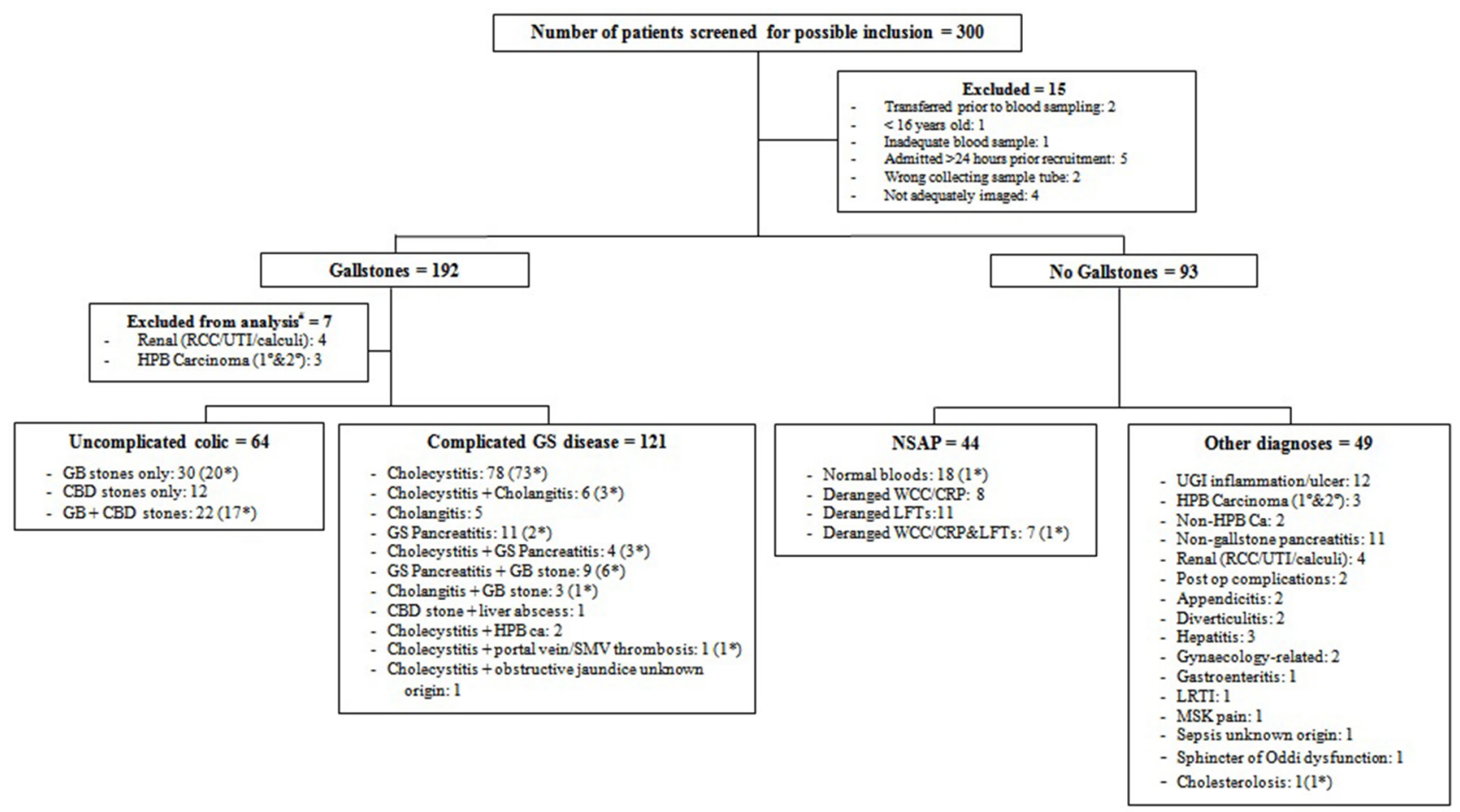

Figure 1 Flow diagram for the main study. The number of patients who underwent cholecystectomy within each group is illustrated in brackets with an asterisk). \#These patients had gallstones as an incidental finding and strong evidence for other pathology to account for symptoms. CBD, common bile duct; CRP, C reactive protein; GB, gall bladder; GS, gallstones; HPB, hepatopancreaticobiliary; LFT, liver function test; LRTI, lower respiratory tract infection; MSK, musculoskeletal; NSAP, nonspecific abdominal pain; RCC, renal cell cancer; SMV, superior mesenteric vein; UGI, upper gastrointestinal; UTI, urinary tract infection; WCC, white cell count. 
Table 1 Demographics of the study cohort and routine blood test results

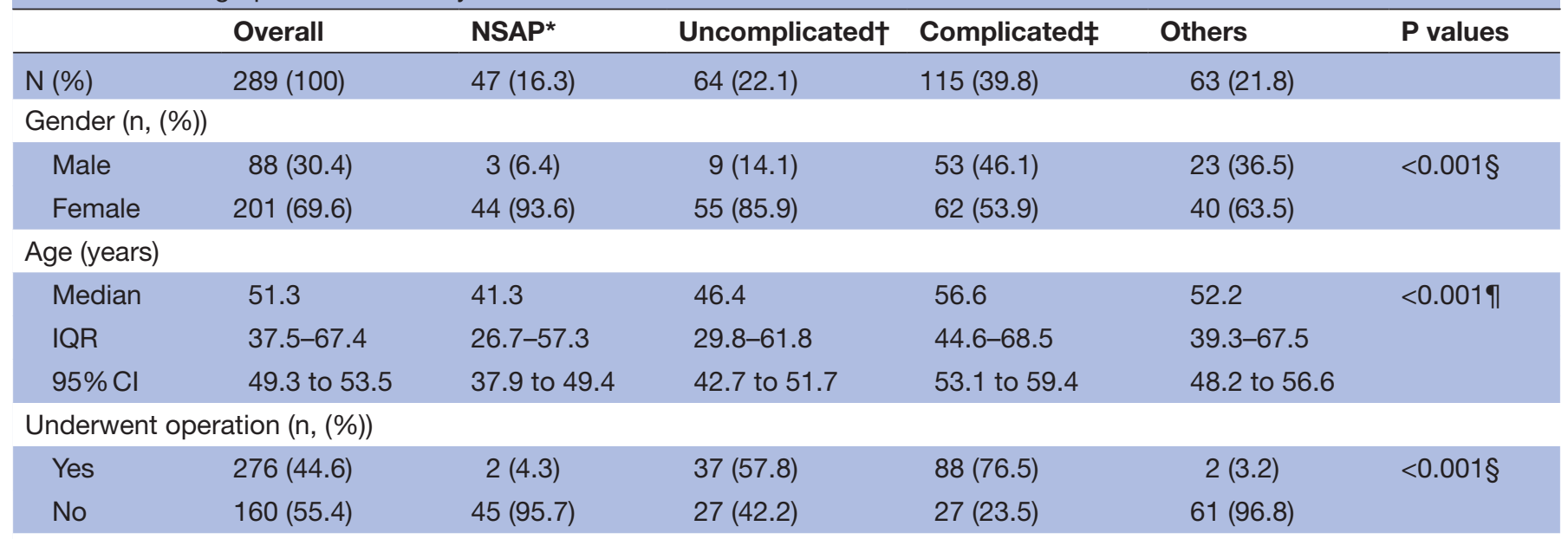

Duration from index presentation to operation (days)

$\begin{array}{lllllr}\text { Median } & 3.0 & 1.5 & 4.0 & 3.0 & 24.0 \\ \text { IQR } & 2.0-5.5 & 1.0 & 2.0-7.5 & 2.0-4.8 & 5.0 \\ 95 \% \mathrm{Cl} & 7.6 \text { to } 17.6 & -4.9 \text { to } 7.9 & 4.1 \text { to } 25.2 & 5.8 \text { to } 17.6 & -217.4 \text { to } 265.4\end{array}$

Total bilirubin $(\mu \mathrm{mol} / \mathrm{L})$

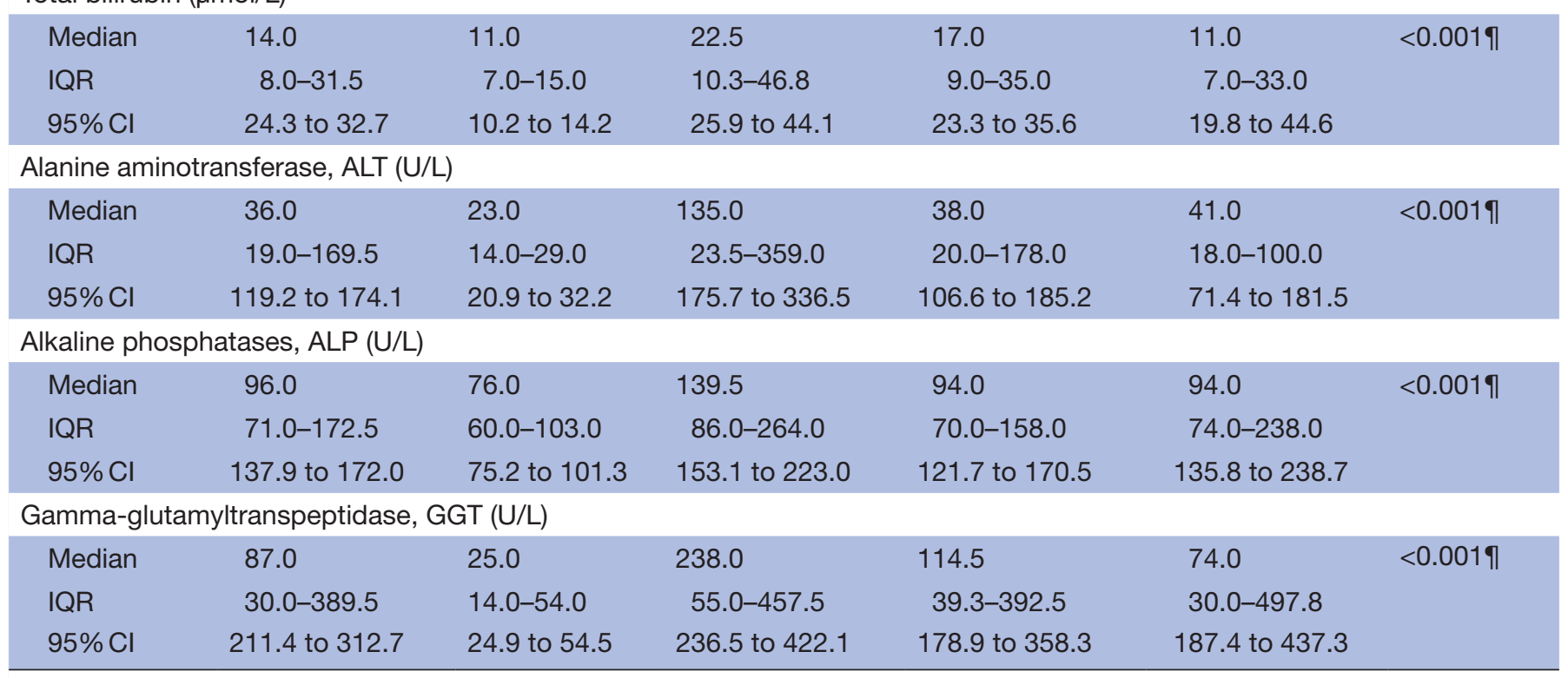

*Non-specific abdominal pain.

†Biliary colic, aseptic choledocholithiasis.

‡Cholecystitis, cholangitis, gallstone pancreatitis.

$\S$ Pearson's $\chi^{2}$ test (for 'gender' and 'underwent operation').

ๆKruskal-Wallis test and Dunn's multiple comparison test (for 'age', 'duration from index presentation to operation', 'total bilirubin', 'ALT',

'ALP' and 'GGT').

NSAP, non-specific abdominal pain.

Pearson's $\chi^{2}$ test was used to examine the association between categorical variables. The Kolmogorov-Smirnov test was used to compare the distribution of non-parametric data against the normal distribution. The Kruskal-Wallis test was used to compare non-parametric variables followed by Dunn's post hoc test for multiple comparisons. 95\% CIs were calculated for all normally distributed continuous variables. Positive predictive value (PPV) and negative predictive value (NPV) were used as the most relevant measures of clinical utility.
Receiver operating characteristic (ROC) curve analyses were plotted for the different patient groups. Areas under the ROC curves were calculated with 95\% CI. Sensitivity, PPV and NPV were obtained at $90 \%$ specificity. Spearman's correlation was used to evaluate the correlation between age and gender in the cohort; linear regression was used to evaluate the effect of age (continuous variable) and gender (categorical variable) on biomarker-level expression (Stepping method criteria; Probability of F-entry 0.05; removal 0.10); backwards logistic 
Table 2 Biomarker concentrations

\begin{tabular}{|c|c|c|c|c|c|c|}
\hline & Overall & NSAP* & Uncomplicated† & Complicated‡ & Others & P values§ \\
\hline \multicolumn{7}{|c|}{ miR-122 ( $\times 10^{4}$ copies $/ \mathrm{mL}$ ) } \\
\hline Median & 1.81 & 0.90 & 5.72 & 2.26 & 1.13 & \multirow[t]{2}{*}{$<0.001$} \\
\hline $95 \% \mathrm{Cl}$ & 6.21 to 16.40 & 4.32 to 26.42 & 9.51 to 22.24 & 1.54 to 22.82 & 2.26 to 8.23 & \\
\hline \multicolumn{7}{|c|}{ HMGB1 (ng/mL) } \\
\hline IQR & $1.34-3.55$ & $1.26-3.50$ & $1.30-4.67$ & $1.44-3.37$ & $1.18-4.16$ & \multirow{2}{*}{0.584} \\
\hline $95 \% \mathrm{Cl}$ & 2.13 to 2.60 & 1.50 to 2.59 & 2.05 to 3.01 & 2.02 to 2.56 & 1.90 to 3.10 & \\
\hline \multicolumn{7}{|c|}{ cck-18 (U/L) } \\
\hline Median & 114.60 & 100.33 & 139.71 & 111.35 & 114.84 & \multirow[t]{2}{*}{0.009} \\
\hline IQR & $87.40-171.53$ & $75.05-135.40$ & $98.15-228.71$ & $87.17-171.83$ & $87.12-181.26$ & \\
\hline Median & 202.99 & 107.08 & 322.55 & 236.34 & 205.54 & \multirow[t]{3}{*}{$<0.001$} \\
\hline IQR & $102.63-450.55$ & $66.39-168.43$ & $133.43-662.04$ & $136.63-429.38$ & $101.81-462.56$ & \\
\hline $95 \% \mathrm{Cl}$ & 170.60 to 237.24 & 78.90 to 149.70 & 202.10 to 479.32 & 184.91 to 304.06 & 151.78 to 325.69 & \\
\hline
\end{tabular}

*Non-specific abdominal pain.

†Biliary colic, aseptic choledocholithiasis.

$\ddagger$ Cholecystitis, cholangitis, gallstone pancreatitis.

§Kruskal-Wallis test and Dunn's multiple comparison test.

cck-18, caspase-cleaved keratin-18; flk-18, full-length keratin-18; HMGB1, high-mobility group box 1; miR-122, microRNA-122; NSAP, non-

specific abdominal pain.

regression (Probability for stepwise entry $\mathrm{p}=0.05$ and removal from the model $\mathrm{p}=0.10$, classification cut-off $0.5,20$ iterations) was used to explore the confounding effect of age (as a continuous variable) and gender (as a categorical variable) on the association between biomarker levels (as continuous variables) and the presence of gallstones, or complicated gallstones (as separate binary dependent variables). All statistical tests were based on a two-sided $\alpha$-value of 0.05 . Statistical analysis was performed using IBM SPSS Statistics V.19.0 (IBM) and $\mathrm{G}^{*}$ Power V.3.1 (Universität Düsseldorf, Germany). Figures were designed, and correlation analyses were carried out using GraphPad Prism V.6.0 (GraphPad Software, La Jolla, California, USA).

\section{RESULTS}

\section{Study population}

A total of 300 patients were screened for possible inclusion in the study. The study flow chart is presented in figure 1. Fifteen patients who had consented to participate in the study were excluded: two were transferred to a different hospital prior obtaining blood samples; one was under 16 years old; five were admitted more than 24 hours prior to recruitment; two patients' blood samples were collected in the wrong sample tube; one blood sample tube did not have the required volume for biomarker testing; four patients did not receive any imaging to determine a cause for their abdominal pain for reasons that we were unable to ascertain, but may have included premature self-discharge. Of the 285 patients included in the study, seven patients with an incidental finding of gallstones were excluded from data analysis as there was strong clinical and radiological evidence for a different pathology to account entirely for their symptoms. Forty-four (15.8\%) patients had NSAP, 64 patients $(23.0 \%)$ had uncomplicated gallstone disease, 121 patients $(43.5 \%)$ had a complicated gallstone disease and 49 patients $(17.7 \%)$ had other non-gallstone-related disease.

The demographics of the study subjects are illustrated in table 1 . There were more females compared with males between the four patient groups $(\mathrm{p}<0.001)$. Patients with uncomplicated gallstone diseases (46.5 years (29.8-61.8)) were significantly younger than patients with complicated diseases (57.4 years $(44.8-68.6) ; \mathrm{p}<0.001)$. Serum alanine aminotransferase, alkaline phosphatase and gamma-glutamyltranspeptidase activities were higher in patients with uncomplicated gallstone diseases compared with complicated diseases.

Of the 278 patients included for data analysis, 129 patients $(46.4 \%)$ underwent a cholecystectomy. There was no difference in the time period from patient's index hospital presentation to the day of operation between patient with (median: 3.0 days $(2.0-5.0)$ ) and without (median: 4.0 days (2.0-7.5)) complications of gallstones $(\mathrm{p}=0.137)$. Interestingly, there was no statistical difference between the two gallstone disease 
Table 3 Biomarker evaluation by receiver operator characteristics

ROC AUC (95\% Cl) Sensitivity (\%) $(95 \% \mathrm{Cl}) \quad$ PPV (\%) NPV (\%) P values*

\begin{tabular}{|c|c|c|c|c|c|}
\hline \multicolumn{6}{|l|}{ miR-122 } \\
\hline Gallstone versus non-gallstone $\dagger$ & 0.66 (0.59 to 0.72$)$ & 16 (10 to 25$)$ & 72.5 & 39.8 & $<0.001$ \\
\hline Uncomplicatedł versus complicated§ & $0.60(0.51$ to 0.69$)$ & 22 (15 to 30$)$ & 56.0 & 67.5 & 0.022 \\
\hline Gallstone versus non-gallstone $†$ & 0.57 (0.50 to 0.64$)$ & 6 (3 to 13$)$ & 50.0 & 7.1 & 0.041 \\
\hline Uncomplicatedł versus complicated§ & 0.59 (0.50 to 0.68$)$ & 14 (8 to 22$)$ & 45.0 & 65.4 & 0.049 \\
\hline Gallstone versus non-gallstone $†$ & $0.63(0.56$ to 0.70$)$ & 20 (13 to 29$)$ & 76.6 & 40.9 & $<0.001$ \\
\hline Uncomplicatedł versus complicated§ & $0.56(0.47$ to 0.65$)$ & 12 (7 to 20$)$ & 42.1 & 65.0 & 0.160 \\
\hline
\end{tabular}

*Kruskal-Wallis test and Dunn's multiple comparison test.

†NSAP and other diseases.

$\ddagger$ ‡iliary colic, aseptic choledocholithiasis.

$\S$ Cholecystitis, cholangitis, gallstone pancreatitis.

AUC, area under the curve; cck-18, caspase-cleaved keratin-18; flk-18, full-length keratin-18; miR, microRNA-122; NPV, negative predictive

value; NSAP, non-specific abdominal pain; PPV, positive predictive value; ROC, receiver operating characteristic.

subgroups (uncomplicated: $\mathrm{n}=11,17.2 \%$ vs complicated: $\mathrm{n}=24,19.8 \%$; $\mathrm{p}=0.664$ ) with regard to $\mathrm{CT}$ and MRCP investigation (uncomplicated: $\mathrm{n}=34,53.1 \%$ vs complicated: $\mathrm{n}=47,38.8 \%$; $\mathrm{p}=0.063$ ) (online supplementary table 1 ).

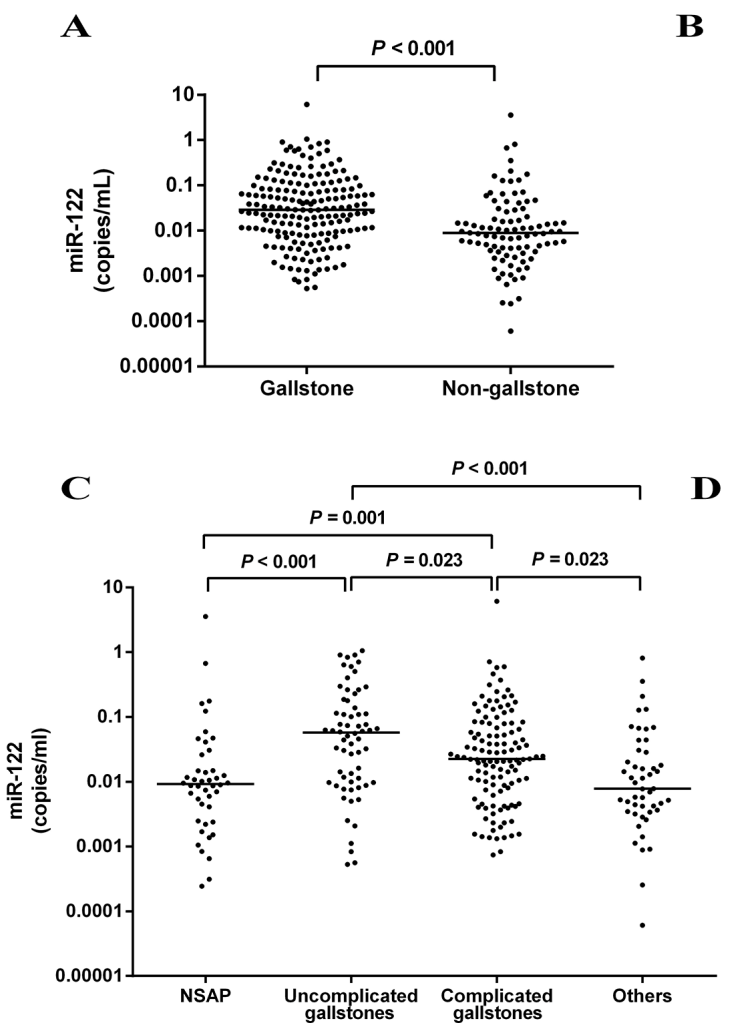

\section{High-mobility group box 1}

There was no significant difference in plasma HMGB1 concentration between patients with and without gallstone disease (gallstone diseases: $2.41 \mathrm{ng} / \mathrm{mL}(1.40-$ 3.53); non-gallstones: $2.41 \mathrm{ng} / \mathrm{mL}(1.26-3.73) ; \mathrm{p}=0.559)$

Figure 2 (A) Plasma microRNA-122 (miR-122) values in gallstone and non-gallstone diseases normalised to miR-39. Each data point represents an individual. The horizontal line in each patient group represents the median value. Statistical significances ( $p$ value) by Kruskal-Wallis test and Dunn's multiple comparison test are shown in the figure for between-group comparison. (B) Receiver operating characteristic (ROC) curve of miR-122 for gallstone versus non-gallstone diseases. (C) miR-122 values for the four main patient groups. (D) ROC curve of miR-122 values for uncomplicated versus complicated gallstone diseases. AUC, area under the curve; NSAP, non-specific abdominal pain; SENS, sensitivity. 
A
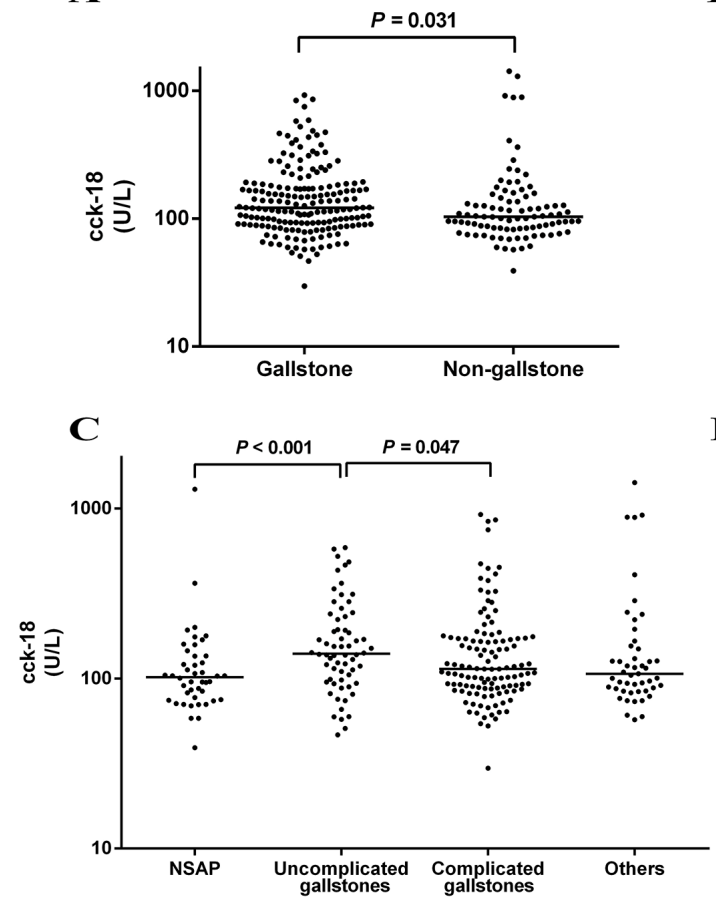

B

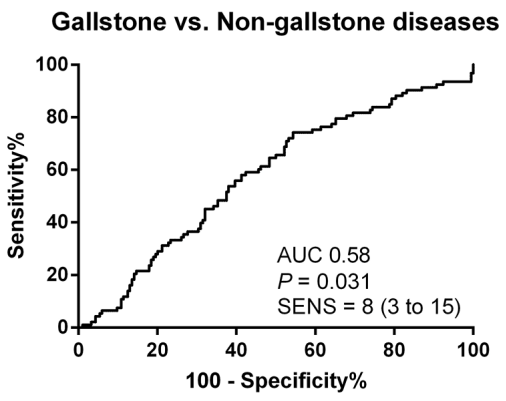

$\mathbf{D}$

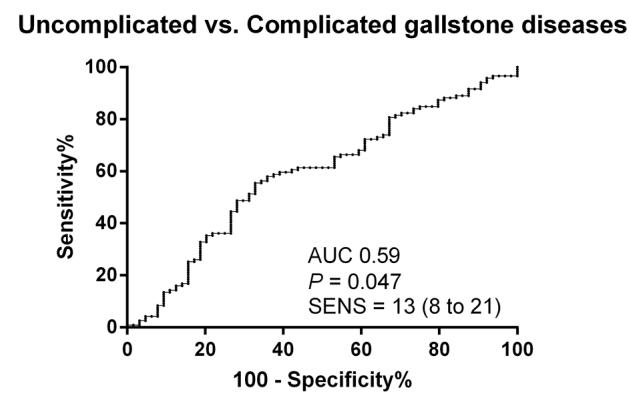

Figure 3 (A) Plasma concentrations of caspase-cleaved keratin-18 (cck-18) protein in gallstone and non-gallstone diseases. Each data point represents an individual. The horizontal line in each patient group represents the median value. Statistical significances ( $p$ value) by Kruskal-Wallis test and Dunn's multiple comparison test are shown in the figure for between-group comparison. (B) Receiver operating characteristic (ROC) curve of cck-18 for gallstone versus non-gallstone diseases. (C) cck18 values for the four main patient groups. (D) ROC curve of cck-18 values for uncomplicated versus complicated gallstone diseases. AUC, area under the curve; NSAP, non-specific abdominal pain; SENS, sensitivity.

or between the four different study groups $(\mathrm{p}=0.964)$ (table 2 and online supplementary figure 1).

\section{microRNA-122}

miR-122 in gallstone diseases and non-gallstone diseases

Circulating miR-122 concentration was higher in patients with gallstone disease than in those without gallstones $\left(2.89 \times 10^{4}\right.$ copies $/ \mathrm{mL}\left(0.89-9.91 \times 10^{4}\right)$ vs $0.90 \times 10^{4}$ copies/ $\left.\mathrm{mL}\left(0.36-2.73 \times 10^{4}\right) ; \mathrm{p}<0.001\right)$ (figure $\left.2 \mathrm{~A}\right)$. ROC curve analysis (figure 2B) of miR-122 for patients with and without gallstone disease produced an area under the curve (AUC) of 0.68. The calculated PPV and NPV at $90 \%$ specificity were $77.5 \%$ and $35.3 \%$, respectively (table 3 ).

miR-122 in uncomplicated and complicated gallstone diseases

Patients with uncomplicated gallstone disease had a significantly higher miR-122 concentration than those with complicated gallstone disease $\left(5.72 \times 10^{4}\right.$ copies $/ \mathrm{mL}$ $\left(0.97-16.85 \times 10^{4}\right)$ vs $2.26 \times 10^{4}$ copies $/ \mathrm{mL}\left(0.78-7.88 \times 10^{4}\right)$; $\mathrm{p}=0.023$ ) (table 2 and figure 2C). ROC analysis (figure 2D) comparing uncomplicated and complicated gallstone diseases yielded an AUC of 0.60 (table 3) (online supplementary figure 2 and supplementary tables 2 and 3 illustrate the gallstone disease subgroups).

\section{Caspase-cleaved keratin-18}

cck-18 in gallstone diseases and non-gallstone diseases

Plasma cck-18 concentration was significantly higher in patients with gallstone disease than those without
(121.20 U/L (89.92-180.42) vs $103.46 \mathrm{U} / \mathrm{L} \quad$ (83.90147.13); $\mathrm{p}=0.031$ ) (figure 3A). A ROC curve analysis of cck-18 in patients with and without gallstones yielded an AUC of 0.58 (figure 3B), with a calculated PPV of $62.5 \%$ and NPV of $32.9 \%$ (table 3 ).

cck-18 in uncomplicated and complicated gallstone diseases cck-18 concentration was higher in patients with uncomplicated (139.71 U/L (98.15-228.71)) gallstone diseases than those with complications (113.60 U/L (87.73171.57)), $\mathrm{p}=0.047$ ) (table 2 and figure 3C). This translated to an AUC of 0.59 (figure 3D) with PPV of $40.0 \%$ and NPV of $66.1 \%$ at $90 \%$ specificity (table 3) (online supplementary figure 3 and supplementary tables 3 and 4 illustrate gallstone disease subgroups).

\section{Full-length keratin-18}

flk-18 in gallstone diseases and non-gallstone diseases

Similarly, flk-18 concentration was significantly higher in patients with gallstone disease than those without (252.38 U/L (137.41-521.27) vs $145.06 \mathrm{U} / \mathrm{L}$ (75.97292.34); $\mathrm{p}<0.001$ ) (figure 4A). A ROC curve analysis (figure 4B) of flk-18 in patients with and without gallstones yielded an AUC of 0.65 (table 3).

flk-18 in uncomplicated and complicated gallstone diseases There was no significant difference in flk-18 concentration between the two subgroups of gallstone diseases $(p=0.154)$ (table 2 and figure 4C,D) (online supplementary figure 4 
A

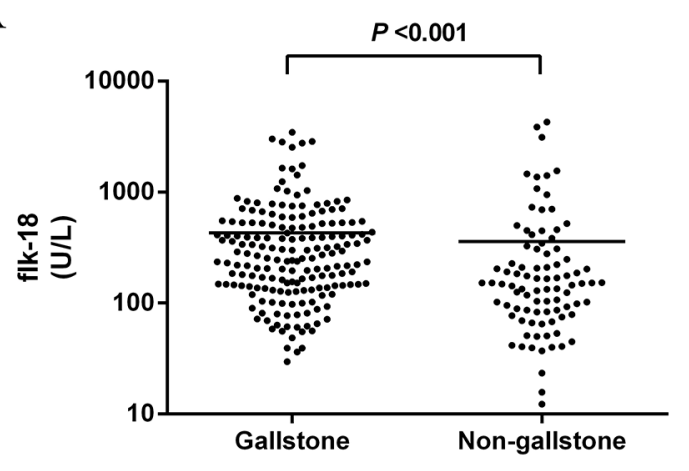

C

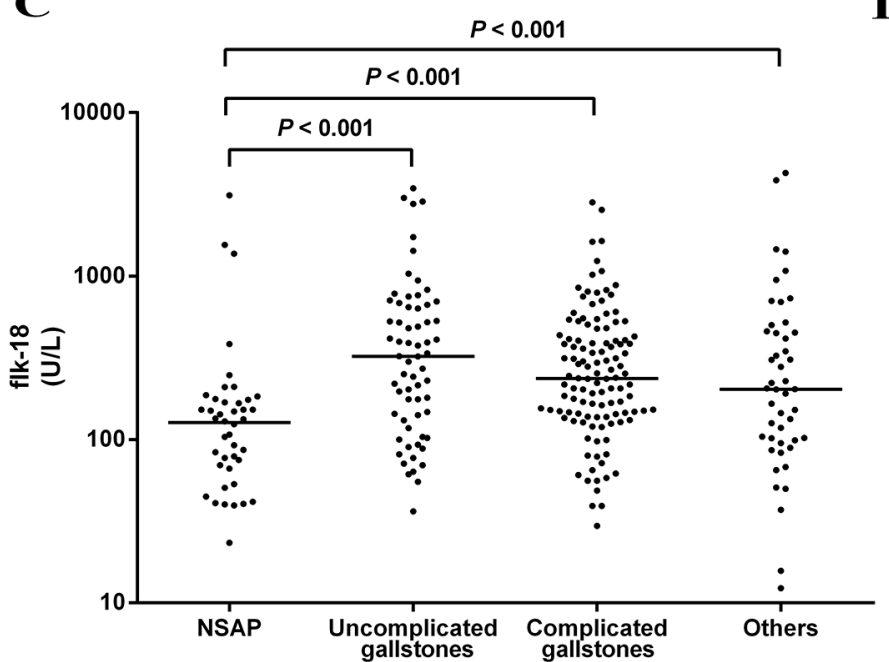

B Gallstone vs. Non-gallstone diseases

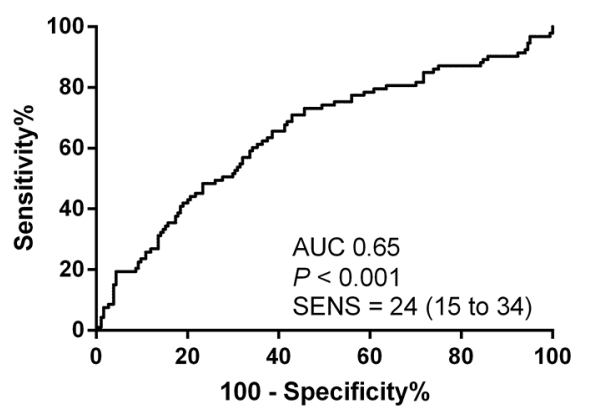

D

Uncomplicated vs. Complicated gallstone diseases

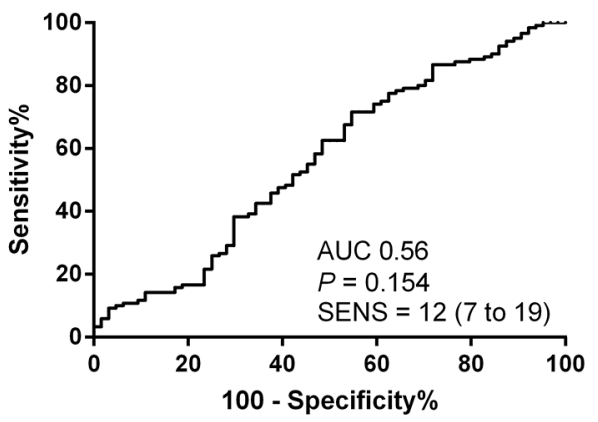

Figure 4 (A) Plasma full-length keratin-18 (flk-18) protein concentrations in gallstone and non-gallstone diseases. Each data point represents an individual. The horizontal line in each patient group represents the median value. Statistical significances ( $p$ value) by Kruskal-Wallis test and Dunn's multiple comparison test are shown in the figure for between-group comparison. (B) Receiver operating characteristic (ROC) curve of flk-18 for gallstone versus non-gallstone diseases. (C) flk-18 concentrations for the four main patient groups. (D) ROC curve of flk-18 values for uncomplicated versus complicated gallstone diseases. AUC, area under the curve; NSAP, non-specific abdominal pain; SENS, sensitivity.

and online supplementary tables 3 and 5 illustrate gallstone disease subgroups).

\section{Effect of age and gender on biomarkers}

Because increasing age and being women exhibited a statistically significant correlation in the cohort (Spearman's $\mathrm{r}=0.285, \mathrm{p}<0.001$ ), we checked whether there was an independent effect of age and gender on any of the biomarker levels by linear regression. Biomarker levels were not independently associated with age or gender (online supplementary table 6). Furthermore, when we examined potential confounding by age and gender on the utility of the biomarker values to detect gallstones by backwards logistic regression, none of age, gender or any biomarker was independently associated with the presence of gallstones (online supplementary table 7 ). For detecting complications of gallstones, using logistic regression, no biomarker was independently associated with the presence of complicated gallstones, but increasing age (OR 1.02; 95\% CI 1.01 to 1.04; $\mathrm{p}=0.002$ ) and female gender (OR 3.10; 95\% CI 1.77 to 5.41; $\mathrm{p}<0.001)$ increased the likelihood of a positive diagnosis of complicated gallstones (online supplementary table 8). Using logistic regression individually for each biomarker did not alter the lack of independent association with the presence of complicated gallstones (online supplementary tables 9-12).

\section{DISCUSSION}

Early identification of individuals with potentially serious complications of gallstones from the denominator pool of all persons presenting with biliary-type symptoms is of translational importance. In this study, we report that plasma miR-122, cck-18 and flk-18 concentrations were significantly higher in patients with gallstone diseases than in non-gallstone diseases. However, the degree of overlap between groups was high, thus detracting from the utility of this approach to accurately stratify on an individual patient basis.

The biomarkers measured in this paper have robust evidence supporting their utility in preclinical models and the clinical setting for the early, sensitive and 
specific identification of hepatocellular injury following paracetamol overdose (an archetypal hepatocyte toxic agent) and also hypoxic hepatitis. Published papers have suggested this panel of biomarkers may also have utility with regard to acute cholestatic pathology induced by gallstones. $^{4-69101516}$

Our finding that plasma miR-122 concentration was increased in patients with gallstones agrees with that of Shifeng et $a l,{ }^{6}$ who compared their disease cohort with 'healthy' control groups. However, their study yielded a much higher AUC on the ROC analysis, 0.93 with $77.4 \%$ sensitivity and $96.4 \%$. The probable explanation for this is that our comparison group was patients admitted acutely under general surgery with a non-gallstone-related diagnosis, who therefore may have had deranged inflammatory markers and/or LFTs due to another pathological process. This demonstrates the importance of clinically relevant control groups in biomarker discovery and validation studies.

In the present study, there was no significant difference in plasma HMGB1 concentration across all comparisons. This is consistent with a study by Shi et at which demonstrated HMGB1 over expression in gallbladder cancers but not in benign GB tissues or cholelithiasis.

Plasma cck-18 and flk-18 were significantly higher in patients with gallstones, in agreement with studies by others $^{1624}$ who examined gallbladder tissue, bile and serum. In our study, patients with cholecystitis were not further subdivided into active/inactive and acute/ chronic subtypes due to sample sizing. Nevertheless, Simopoulos et $a l^{16}$ demonstrated no difference in total k-18 and cck-18 between active and inactive chronic cholecystitis.

We recognise some important limitations in this study. This was a pilot study to determine whether there is a signal of biomarker utility in this context of use to take forward into larger, multicentre studies. We believe that the results presented in this paper are clear in their failure to support utility with regard to patient stratification at hospital presentation. As demonstrated in our analysis, both age and gender are confounding factors in the association of biomarker concentrations with gallstone disease. Serial patient blood sampling for biomarker analyses may have also been useful to determine the true nature of biomarker concentration changes during the disease progress. However, should larger studies be able to show a much stronger association between these biomarkers and the complications of gallstones, these biomarkers could be used as diagnostic aids especially when combined with the current laboratory and imaging investigations available.

In conclusion, miR-122 and keratin-18 plasma concentrations are elevated in patients with gallstones. However, this result is confounded by the association between biomarker concentrations, age and gender. In this pilot study, miR-122 and keratin-18 were not sufficiently discriminatory to be progressed as clinically useful biomarkers in this context.
Author affiliations

${ }^{1}$ Clinical Surgery, School of Clinical Sciences and Community Health, University of Edinburgh, Edinburgh, UK

${ }^{2}$ BHF Centre for Cardiovascular Science, University of Edinburgh, Edinburgh, UK ${ }^{3}$ MRC Centre for Drug Safety Science, University of Liverpool, Liverpool, UK ${ }^{4} \mathrm{MRC}$ Centre for Inflammation Research, Queen's Medical Research Institute, University of Edinburgh, Edinburgh, UK

${ }^{5}$ General Surgery, Royal Infirmary of Edinburgh, NHS Lothian, Edinburgh, UK

Acknowledgements We are grateful for the expert assistance of the Edinburgh Clinical Research Facility and Kolamunnage Dona, Biomedical Statistician, University of Liverpool, UK.

Collaborators Edinburgh Emergency Surgery Study Group, Royal Infirmary of Edinburgh, NHS Lothian: Graeme Couper, Christopher Deans, Gavin G P Browning, Anna M Paisley, Bruce Tulloh, Richard J E Skipworth, Simon Paterson-Brown, Rajan Ravindran, Andrew de Beaux, ljeoma Azodo.

Contributors FT: designed study; acquired, analysed and interpreted data; drafted and revised paper including the final version to be published; agreed to be accountable for all aspects of the work. BV, JDL and DJA: analysed and interpreted data; revised paper including the final version to be published; agreed to be accountable for all aspects of the work. JWD and DJM: designed study; analysed and interpreted data; revised paper including the final version to be published; agreed to be accountable for all aspects of the work.

Funding This work was supported by the Edinburgh and Lothians Health Foundation. BV was supported by an NC3Rs PhD Studentship (NC/K001485/1).

Competing interests None declared.

Patient consent Obtained.

Ethics approval This study was assessed by South East Scotland Research Ethics Service of National Health Service (NHS) Lothian and was given ethical approval under the terms of the Governance Arrangements for Research Ethics Committees (Harmonised Edition). Lothian R\&D project number 2014/0224; REC number 14/ $\mathrm{EM} / 0211$.

Provenance and peer review Not commissioned; externally peer reviewed.

Data sharing statement There are no additional unpublished data from the study.

Open Access This is an Open Access article distributed in accordance with the Creative Commons Attribution Non Commercial (CC BY-NC 4.0) license, which permits others to distribute, remix, adapt, build upon this work non-commercially, and license their derivative works on different terms, provided the original work is properly cited and the use is non-commercial. See: http://creativecommons.org/ licenses/by-nc/4.0/

(c) Article author(s) (or their employer(s) unless otherwise stated in the text of the article) 2018. All rights reserved. No commercial use is permitted unless otherwise expressly granted.

\section{REFERENCES}

1. Portincasa P, Moschetta A, Palasciano G, et al. Cholesterol gallstone disease. Lancet 2006;368:230-9.

2. NHS Digital. Hospital Episode Statistics, Admitted Patient Care - England, 2011-12 [NS]. http://content.digital.nhs.uk/ searchcatalogue?productid $=9161 \& q=$ gallbladder\&infotype $=$ $0 \% 2 \mathrm{fOfficial}+$ statistics \&sort=Relevance\&size=50\&page=1\#top (assessed 15 Jun 2017).

3. Borzellino G, Steccanella F, Mantovani W, et al. Predictive factors for the diagnosis of severe acute cholecystitis in an emergency setting. Surg Endosc 2013;27:3388-95.

4. Dear JW, Antoine DJ, Starkey-Lewis P, et al. Early detection of paracetamol toxicity using circulating liver microRNA and markers of cell necrosis. Br J Clin Pharmacol 2014;77:904-5.

5. Antoine DJ, Jenkins RE, Dear JW, et al. Molecular forms of HMGB1 and keratin-18 as mechanistic biomarkers for mode of cell death and prognosis during clinical acetaminophen hepatotoxicity. $J$ Hepatol 2012;56:1070-9.

6. Shifeng H, Danni W, Pu C, et al. Circulating liver-specific miR-122 as a novel potential biomarker for diagnosis of cholestatic liver injury. PLoS One 2013;8:e73133.

7. Song KH, Li T, Owsley E, et al. A putative role of micro RNA in regulation of cholesterol 7alpha-hydroxylase expression in human hepatocytes. J Lipid Res 2010;51:2223-33. 
8. Li T, Francl JM, Boehme S, et al. Regulation of cholesterol and bile acid homeostasis by the cholesterol $7 \alpha$-hydroxylase/steroid response element-binding protein 2/microRNA-33a axis in mice. Hepatology 2013;58:1111-21.

9. Shi Z, Huang Q, Chen J, et al. Correlation of HMGB1 expression to progression and poor prognosis of adenocarcinoma and squamous cell/adenosquamous carcinoma of gallbladder. Am J Transl Res 2015;7.

10. Antoine DJ, Williams DP, Kipar A, et al. High-mobility group box-1 protein and keratin-18, circulating serum proteins informative of acetaminophen-induced necrosis and apoptosis in vivo. Toxicol Sci 2009;112:521-31.

11. Chu PG, Weiss LM. Keratin expression in human tissues and neoplasms. Histopathology 2002;40:403-39.

12. Wilkins BJ, Gong W, Pack M. A novel keratin18 promoter that drives reporter gene expression in the intrahepatic and extrahepatic biliary system allows isolation of cell-type specific transcripts from zebrafish liver. Gene Expr Patterns 2014;14:62-8.

13. Caulín C, Salvesen GS, Oshima RG. Caspase cleavage of keratin 18 and reorganization of intermediate filaments during epithelial cell apoptosis. J Cell Biol 1997;138:1379-94.

14. Lorente L, Martín MM, González-Rivero AF, et al. Serum levels of caspase-cleaved cytokeratin-18 and mortality are associated in severe septic patients: pilot study. PLoS One 2014;9:e109618.

15. Woolbright BL, Antoine DJ, Jenkins RE, et al. Plasma biomarkers of liver injury and inflammation demonstrate a lack of apoptosis during obstructive cholestasis in mice. Toxicol Appl Pharmacol 2013;273:524-31.

16. Simopoulos C, Tsaroucha AK, Asimakopoulos B, et al. Total and caspase-cleaved cytokeratin 18 in chronic cholecystitis: a prospective study. BMC Gastroenterol 2008;8:14.
17. Qiagen. miRNeasy Serum/Plasma Handbook: miRNeasy Serum/ Plasma Kit For purification of total RNA, including miRNA, from animal and human plasma and serum; miRNeasy Serum/Plasma Spike-In Control; For normalization of miRNA purification from serum or plasma. Qiagen: Sample and Assay Technologies. $2012 \mathrm{http}: / /$ www.qiagen.com/aw/resources/download.aspx?id=710c0168-e408408b-95af-91df5b5b1dd6\&lang=en (assessed 15 Jun 2017).

18. Applied Biosystems. TaqMan Small RNA Assays (TaqMan microRNA assays; TaqMan siRNA assays; Custom TaqMan small RNA assays) Protocol. Applied Biosystems by Life Technologies. [Online]. 2011 https://tools.lifetechnologies.com/content/sfs/manuals/cms_042167. pdf (assessed 15 Jun 2017).

19. Livak KJ, Schmittgen TD. Analysis of relative gene expression data using real-time quantitative PCR and the 2(-Delta Delta C(T)) Method. Methods 2001;25:402-8.

20. SHINO-TEST Corporation. HMGB1 ELISA Kit: Assay Principle. [Online]. 2017 http://www.hmgb1.net/en/products/elisa.html (assessed 15 Jun 2017).

21. IBL International. HMGB1 ELISA. [Online]. $2011 \mathrm{http} / / /$ novamedline. com/downloads/instructions/en/ST51011.pdf (assessed 15 Jun 2017).

22. PEVIVA Products. M65 ELISA. [Online]. 2014 http://diapharma.com/ wp-content/uploads/2017/03/P10020 Peviva M65 ELISA 2014-1219_Rev90097_insert.pdf (assessed 15 Jun 2017).

23. Antoine DJ, Dear JW, Lewis PS, et al. Mechanistic biomarkers provide early and sensitive detection of acetaminophen-induced acute liver injury at first presentation to hospital. Hepatology 2013;58:777-87.

24. Kasprzak A, Malkowski W, Seraszek A, et al. Cytokeratin 8 and 18 tissue expression in gallbladder mucosa of patients with cholelithiasis. Pol J Pathol 2011;62:241-9. 